\title{
PIECEWISE SMOOTH SIGNAL DENOISING VIA PRINCIPAL CURVE PROJECTIONS
}

\author{
Umut Ozertem*, Deniz Erdogmus ${ }^{\dagger}$, Orhan Arikan * \\ *Yahoo! Inc. Santa Clara CA, 95054, \\ ${ }^{\dagger}$ CSEE Dept. Oregon Health and Science University, Portland OR, 97329 \\ ${ }^{\star}$ Dept. of Electrical and Electronics Engineering, Bilkent University, TR-06800 Bilkent, Ankara
}

\begin{abstract}
One common problem in signal denoising is that if the signal has a blocky, in other words a piecewise-smooth structure, the denoised signal may suffer from oversmoothed discontinuities or exhibit artifacts very similar to Gibbs phenomenon. In the literature, total variation methods and some modifications on the signal reconstructions based on wavelet coefficients are proposed to overcome these problems. We take a novel approach by introducing principal curve projections as an artifact-free signal denoising filter alternative. The proposed approach leads to a nonparametric denoising algorithm that does not lead to Gibbs effect or so-called staircase type unnatural artifacts in the denoised signal.
\end{abstract}

\section{INTRODUCTION}

Following the additive noise model, signal denoising problem can be defined as extracting the meaningful part of the data from the observed signal by subtracting or suppressing noise. Although in most of the scenarios this intuitively refers to reconstruction of the original signal from the observed signal that is contaminated with noise; in general, the vagueness of meaningful part in the problem definition here is indeed intended depending on what the problem specific meaningful information is.

Under the known noise model assumption, optimal filtering in frequency domain is a well established topic [1]. Frequency domain filtering is conceptually simple, easily analyzable, and computationally very inexpensive. However, the main drawback of this approach is obvious if the signal and the noise are not separable in the frequency domain. Practically, in frequency domain filtering one should compromise the discontinuities in the signal, since they will be smoothed along with the noise.

As well as traditional frequency domain filtering techniques, current signal denoising techniques include wavelet transform and total variation based noise removal algorithms. These techniques stem from the idea of preserving the high

This work is partially supported by NSF ECS-0524835, and NSFECS0622239. frequency components, namely discontinuities, of the signal while suppressing noise. Since piecewise smooth signal structure is typical in visual images, total variation based noise removal is first introduced in image denoising $[2,3]$. Later, Vogel proposed a fixed-point iterative scheme to solve the total variation penalized least squares problem [4].

Another way to achieve discontinuity preserving denoising is to utilize discrete wavelet transform as an orthogonal basis decomposition. This method is based on decomposing the signal into orthogonal wavelet basis, perform either soft or hard thresholding to wavelet coefficients, and transform the data to the time/spatial domain by inverse discrete wavelet transform. Note that any orthogonal decomposition of the signal can be used similarly for denoising. In such techniques the performance of the denoising is typically measured by the rate of decay of the basis coefficients sorted in the magnitude. In the context of piecewise smooth signal denoising, wavelet decomposition is known to be superior to other widely used choices like Fourier and cosine decompositions [5]. Earlier techniques based on hard thresholding of the wavelet coefficients suffer from Gibbs effect like artifacts around the discontinuities. This drawback is handled by soft thresholding of this wavelet coefficients [6], and later by applying previously mentioned total variation based approaches in wavelet domain analysis [7].

Principal curves are defined by Hastie and Stuetzle [8] as "self-consistent finite length smooth curves passing from the middle of data." The literature on principal curves is dense in algorithm design, but there is not much work on the theoretical side. We recently proposed another definition for principal curves, which describes the principal curve in terms of the gradient and the Hessian of the data probability density $[9,10]$ that yields constrained maximum likelihood type algorithms. This definition stems from a differential geometric approach. In this paper, we utilize our recently proposed principal curve projections to implement a data-driven nonparametric nonlinear filter for artifact-free piecewise continuous signal denoising. The resulting filter is based on the kernel density estimate (KDE) of the data, where parametric variants are possible and conceptually very similar. 


\section{PRINCIPAL CURVES}

We define the principal curve as follows: "a point in the data feature space is on the principal curve if and only if the gradient of the pdf is parallel to one of the eigenvectors of the Hessian and the remaining eigenvectors have negative eigenvalues" $[9,10]$. In other words, the principal curve is essentially the ridge of the pdf.

Although the details of the principal curve derivation is omitted due to restricted space, we start with a simple illustration of the concept here to motivate our proposition. Consider the simple illustration in Figure 1. To find the principal curve, we start with the density estimate of the data. Figure 1a shows the dataset (blue) along with the principal curve projections of all data samples (green). This is exactly where the gradient of the pdf is parallel with one of the eigenvectors of the Hessian of the pdf. More intuitively, the principal curve can also be thought as the ridge of the pdf. Underlying probability density along with the same principal curve is presented in Figure 1b, again for the same dataset.

Defining the principal curve in terms of the data pdf allows us to leave all regularization constraints to the density estimation step. This idea, in fact, is central to our approach of connecting the known problems in the principal curve fitting research to well-studied results in the density estimation literature. In the next section, as we present our implementation, we will present brief examples of these natural connections in the context of kernel density estimation.

\section{PIECEWISE SMOOTH SIGNAL DENOISING VIA PRINCIPAL CURVE PROJECTIONS}

This section will present the details of the proposed principal curve denoising filter, as well as the selection of the kernel bandwidth, which is a critical step in the implementation. To utilize principal curve projections, one should start with translating the signal into a suitable feature space. The most intuitive selection is to use the samples of the noisy signal itself along with the associated time indices. Assuming that the observed signal $x(t)$ is the original signal buried in additive noise that is, $x(t)=s(t)+n(t)$, this yields

$$
\mathbf{x}_{i}=\left[\begin{array}{c}
x\left(t_{i}\right) \\
t_{i}
\end{array}\right], \quad t_{1}<\ldots<t_{N}
$$

where $t_{i}$ denote the sampling times. At this point, we assume that the original signal $s(t)$ is sampled at least at the Nyquist frequency. Sampling and reconstruction are well established topics in signal processing, the details of which will are out of the scope of this paper. Also note that, as long as it preserves a blockwise continuous nature, the rest of the paper does not depend on how $\mathbf{x}$ is constructed. Using time

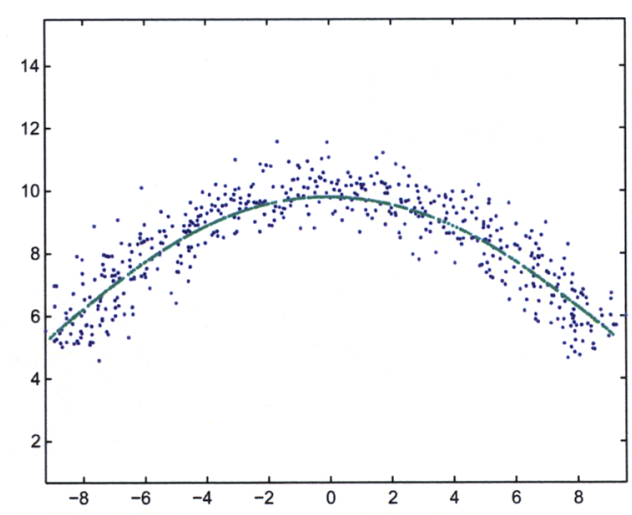

(a) curve dataset and its principal curve

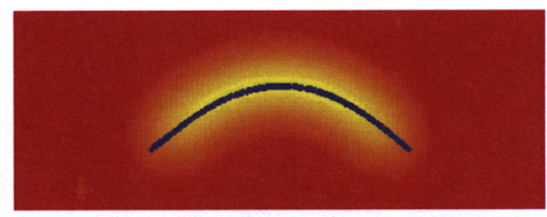

(b) KDE of the curve dataset and with the principal curve

Fig. 1. A simple illustration of the principal curve concept

delayed versions of the observed signal or any other representation of it based on orthogonal decompositions can also be used. For now, we leave this further investigation as future work and focus on the simple feature construct given in (1).

Figure 2 depicts an illustrative piecewise smooth signal with its noisy version as well as the kernel density estimate of the noisy signal. Now consider the kernel density estimate of the noisy signal which is given by

$$
p(\mathbf{x})=\sum_{i=1}^{N} K_{\Sigma_{i}}\left(\mathbf{x}-\mathbf{x}_{i}\right)
$$

where $\Sigma_{i}$ denotes the covariance of the kernel function for the $i^{\text {th }}$ sample ${ }^{l}$. Note that the density estimate of $\mathbf{x}$ in the vicinity of discontinuities is not effected much by the samples on the opposite side of the discontinuity. Although these are close -or maybe even subsequent- samples along the time axis, the discontinuity in signal value makes them sufficiently distant in the $\mathbf{x}$ space. For unimodal additive noise distributions (as in the case of additive Gaussian noise)

\footnotetext{
${ }^{1}$ This density estimate is given for the most general case of datadependent variable bandwidth KDE. For fixed bandwidth version, one can just drop this data dependency.
} 


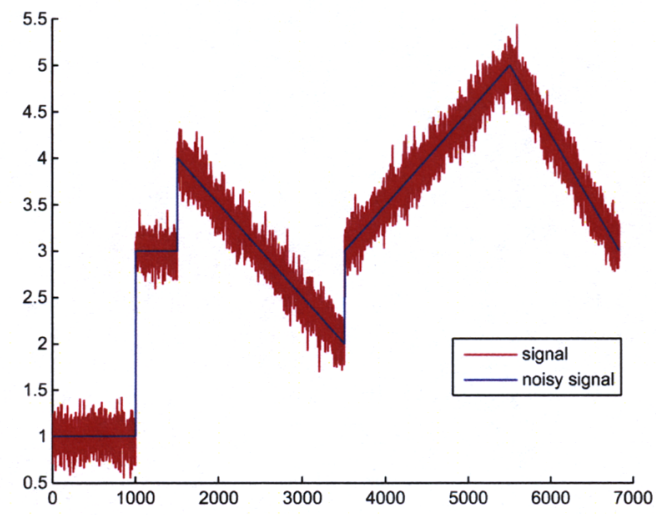

(a) noisy and noiseless signals in time domain

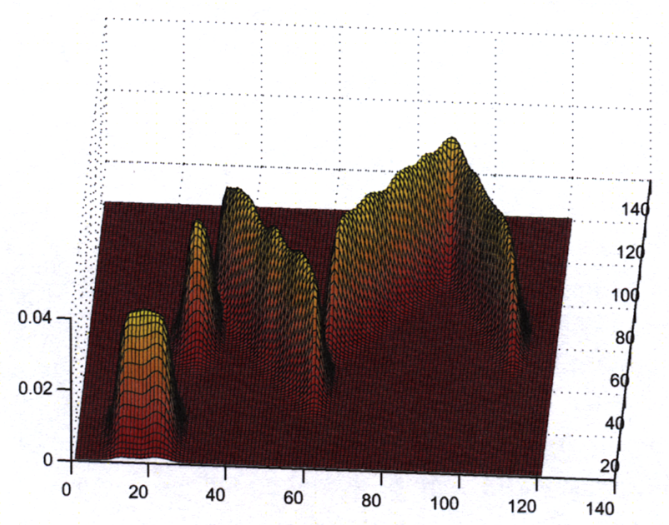

(b) KDE of the noisy signal in $\mathbf{x}$ space

Fig. 2. The underlying noiseless signal and its noisy counterpart

the noiseless signal will most likely be a smoother signal passing through the middle of the observed noisy samples. Hence, principal curve naturally fits into the context of artifact free signal denoising of piecewise smooth signals.

As we proposed in earlier publications $[9,10]$, principal curve projection can be achieved by a likelihood maximization in a constrained space, and our earlier proposed algorithms can directly be used to identify the principal curve. However, particularly for this denoising application, we have a much easier scenario due to the following:

1. Only the samples of the principal curve at time indices $t_{1}<\ldots<t_{N}$ are sufficient. Higher time resolution or seeking for the portion of the principal curve that lies outside the given time interval is unnecessary.

2. Unlike the general case of random vectors, the second dimension, which represents the time indices of signal samples is deterministic; in the case of uniform sampling, we can model this density as being uniform for theoretical analysis.

One can select the initialization of the algorithm and the constrained space of the projection using these two simplifications. At this point, starting from the data samples themselves, and selecting the constrained space as the $t=t_{i}$ for each data sample is our choice for the two following reasons, under the assumption of a unimodal zero mean noise density:

1. Selecting constrained space orthogonal to time index guarantees that there is only one denoised signal value at all time indices.

2. One important observation here is that at the peak of the pdf in each constrained space $t=t_{i}$ is very close to principal curve.

With the above selections of the initialization and the constrained space, the principal curve projection turns out to be as simple as evaluating the mean shift update and projecting it onto the first dimension - the vertical axis in Figure 2a. Although it is close, the optimizer of the algorithm is not on the principal curve. Therefore, we use the SCMS algorithm [10] to project these points onto the principal curve, and assign the signal values as the denoised signal by keeping the time indices the same. We skip the derivation of SCMS here, yet we still provide the details of implementation in Table 1, steps 5-10.

Mean shift is a very commonly used iterative procedure that maps the data points to the corresponding peak of the probability density, where the gradient is equal to zero and all eigenvalues of the Hessian are non-positive [11]. Particularly for a Gaussian kernel function with fixed bandwidth, taking the derivative of (2) with respect to $x$, and equating it to zero, one obtains

$$
\begin{gathered}
p(\mathbf{x})=\sum_{i=1}^{N} G_{\sum_{i}}\left(\mathbf{x}-\mathbf{x}_{i}\right) \\
\mathbf{x} \sum_{i=1}^{N} \Sigma_{i}^{-1} G_{\Sigma_{i}}\left(\mathbf{x}-\mathbf{x}_{i}\right)-\sum_{i=1}^{N} \mathbf{x}_{i} \Sigma_{i}^{-1} G_{\sigma}\left(\mathbf{x}-\mathbf{x}_{i}\right)=0
\end{gathered}
$$

Reorganizing terms and solving for $\mathbf{x}$ yields the well-known mean shift update [11]

$\mathbf{x} \leftarrow \mathbf{m}(\mathbf{x})=\left(\sum_{i=1}^{N} \Sigma_{i}^{-1} G_{\Sigma_{i}}\left(\mathbf{x}-\mathbf{x}_{i}\right)\right)^{-1} \sum_{i=1}^{N} \Sigma_{i}^{-1} G_{\Sigma_{i}}\left(\mathbf{x}-\mathbf{x}_{i}\right) \mathbf{x}_{i}$

Finally, to achieve the desired projection, at each iteration the update in the second dimension has to be removed. This projection has a time complexity of $O(N)$ per sample per iteration. Table 1 briefly presents the overall algorithm. 


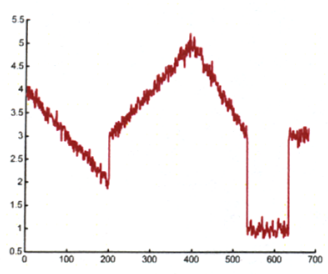

(a)

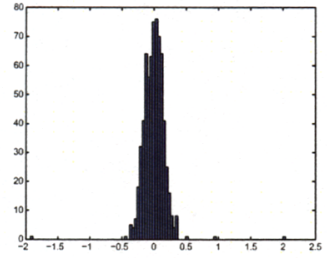

(b)

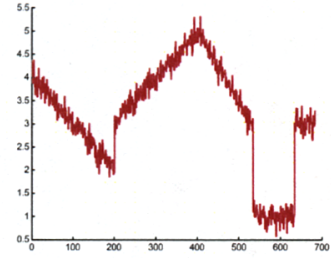

(c)

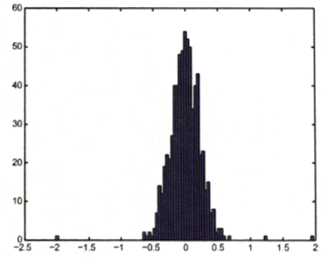

(d)

Fig. 3. Two realizations of the same piecewise smooth signal for two different SNR levels are shown in (a) and (b), along with their time difference histograms in (c) and (d).

Table 1. Principal curve signal denoising

1. Build the feature space $\mathbf{x}$, select the kernel bandwidth $\sigma$ of the Gaussian kernel.

2. Evaluate the mean shift update using (4).

3. Project the mean shift update to $\left[\begin{array}{l}1 \\ 0\end{array}\right]$, so that the mean shift procedure remains in the constrained space.

4. If convergence not achieved, go to step 2 , if convergence is achieved go to the next step.

5. For every trajectory evaluate the mean shift update in (4).

6. Evaluate the gradient, the Hessian, and perform the eigendecomposition of $\boldsymbol{\Sigma}^{-1}(\mathbf{x}(k))=\mathbf{V} \Gamma \mathrm{V}$.

7. Let $\mathbf{v}$ be the leading eigenvector of $\Sigma^{-1}$.

8. $\tilde{\mathbf{x}}=\mathbf{v \mathbf { v } ^ { T }} \mathbf{m}(\mathbf{x}(k))$

9. If $\left|\mathbf{g}^{T} \mathbf{H g}\right| /\|\mathbf{g}\|\|\mathbf{H g}\|>$ threshold then stop, else $\mathbf{x}(k+1) \leftarrow \tilde{\mathbf{x}}$.

10. If convergence is not achieved, increment $k$ and go to step 6.

\subsection{Selection of the Kernel Bandwidth}

Selecting the bandwidth of the Gaussian kernel is a very important step in the implementation that severely affects the performance of the algorithm. Fortunately, literature on density estimation is rich on how to select the kernel width, and methods in the literature extend from simple heuristics to more technically sound approaches like maximum likelihood $[12,13,14,15,16]$.

All above mentioned techniques are general purpose kernel bandwidth selection methods that blindly approach the data. Particularly for this problem, one can also select the kernel bandwidth by considering the actual physical meaning of the data. If the amount of noise and the amount of fluctuations at the discontinuities are at different scales, kernel bandwidth selection can be achieved by observing probability density of the time difference of the signal. Figure 3 presents two realizations of a piecewise smooth signal at different noise levels. Although this may not be the general case for all noise levels, note that the noise distribution and discontinuities are clearly identifiable in this difference histogram for the presented signals, where the difference values at the discontinuities seem like outliers of the Gaussian noise distribution.

\section{EXPERIMENTAL RESULTS}

This section presents the performance of the proposed denoising algorithm. To be able to control the SNR level in the experiments, synthetic examples are used, and denoising performance is reported for different SNR values. Another important point is, of course, the performance around the discontinuities. As well as quantitative results, we present the actual signal plots to provide a visual comparison of the noisy and denoised signals at different SNR levels. At the discontinuities, principal curve denoising does not yield any pseudo-Gibbs artifact effects like discrete wavelet denoising techniques, or any oversmoothing like frequency domain filtering. As one can observe from the denoising on the ramp-signal components, principal curve denoising also 


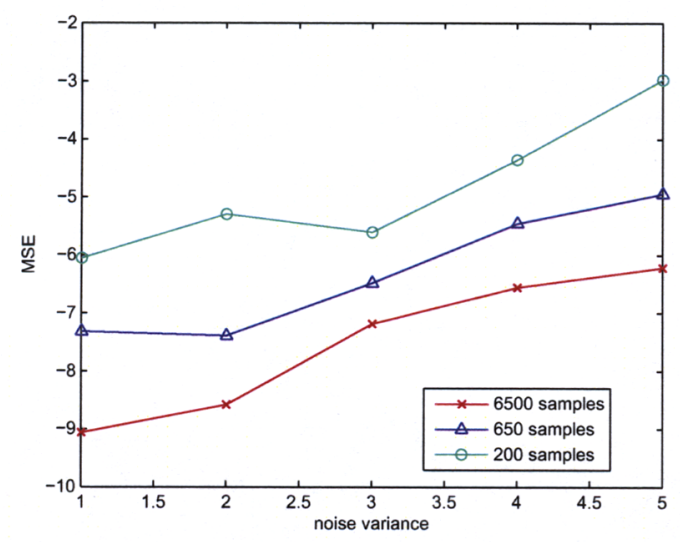

Fig. 4. MSE between the noiseless signal and output of the denoising filter for different SNR levels

does not yield any staircase type effects like total variation based methods.

Figure 4 presents the denoising results for the noiseless signal presented in Figure 2 (blue) for different SNR levels. We measure the performance MSE between the output of principal curve denoising and the underlying noiseless signal. Here we also compare the number of samples used in the principal curve estimation. The principal curve projection depends on the accuracy of the underlying density estimate. As the statistical variance of the density estimate increases in low sampling rates, and the performance of the principal curve denoising decreases.

For the same signals of different SNR and sampling rates, which are compared in Figure 4, Figure 5 presents the noisy and denoised time signal pairs. For all above experiments, we use the maximum likelihood kernel size for each signal. So, we learn the kernel bandwidth from the signal directly, and use a different kernel bandwidth for each realization of the signal. Maximum likelihood training automatically adapts the kernel bandwidth according to the noise level of the data, and evaluating the maximum likelihood kernel bandwidth is a well-known topic and details are omitted here [12].

As a final remark, note that we are not presenting any comparisons with the other principal curve approaches in the literature. Since, by definition, they are looking for smooth curves, earlier principal curve algorithms are not suitable for piecewise smooth signals, and oversmoothing on the discontinuities would be unavoidable.

\section{CONCLUSIONS}

In this paper, we propose to use principal curves for artifactfree denoising of piecewise smooth signals. The proposed denoising filter can successfully preserve the sharp discontinuities in the data under the additive unimodal noise assumption, without introducing any smoothing, high frequency Gibbs effect like artifacts or staircase type effects.

The proposed scheme allows one to translate common problems in piecewise smooth signal denoising into selection of the kernel function. For example, as opposed to earlier methods in the piecewise-smooth signal denoising that are based on total variation or discrete wavelet transform, principal curve based denoising is much easier to build an online counterpart just by selecting a finite support kernel function. For a KDE built using a kernel function of online support, the subset of signal samples that affect the pdf on a particular point of interest are clearly given, yielding an online version of the algorithm directly. Adapting the principal curve denoising for nonstationary or data-dependent noise distributions is also straightforward. With the current implementation, it is computationally more expensive than earlier methods in the literature. At this point, KDE with finite support kernel will save a lot of computational effort, which can bring the computational cost up to linear time complexity. All these are left as future prospects to investigate.

\section{REFERENCES}

[1] Simon Haykin, Adaptive filter theory (3rd ed.), Prentice-Hall, Inc., Upper Saddle River, NJ, USA, 1996.

[2] Leonid I. Rudin, Stanley Osher, and Emad Fatemi, "Nonlinear total variation based noise removal algorithms," Phys. D, vol. 60, no. 1-4, pp. 259-268, 1992.

[3] David C. Dobson and Fadil Santosa, "Recovery of blocky images from noisy and blurred data," SIAM Journal on Applied Mathematics, vol. 56, no. 4, pp. 1181-1198, 1996.

[4] C. R. Vogel and M. E. Oman, "Iterative methods for total variation denoising," SIAM Journal on Scientific Computing, vol. 17, no. 1, pp. 227-238, 1996.

[5] Albert Cohen and Jean-Pierre D'Ales, "Nonlinear approximation of random functions," SIAM Journal on Applied Mathematics, vol. 57, no. 2, pp. 518-540, 1997.

[6] David L. Donoho, "De-noising by soft-thresholding," IEEE Transactions on Information Theory, vol. 41, no. 3, pp. 613-627, 1995.

[7] Sylvain Durand and Jacques Froment, "Reconstruction of wavelet coefficients using total variation minimization," SIAM J. Sci. Comput., vol. 24, no. 5, pp. 1754-1767, 2002. 


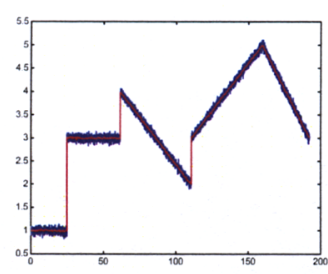

(a)

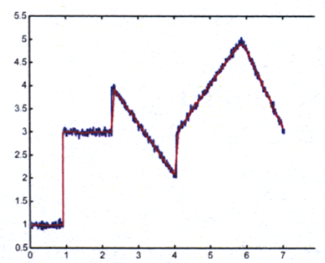

(f)

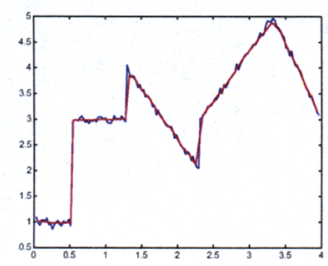

(k)

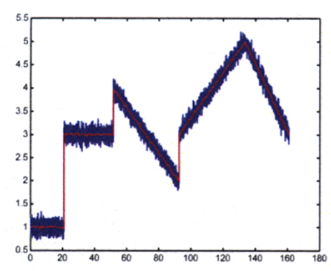

(b)

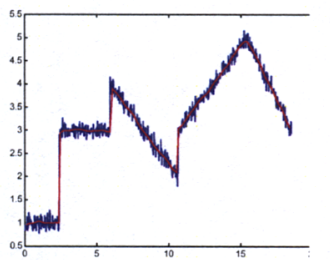

(g)

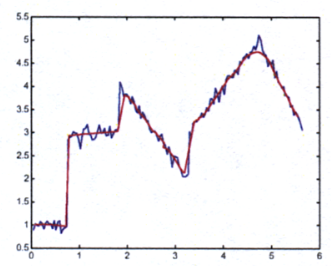

(1)

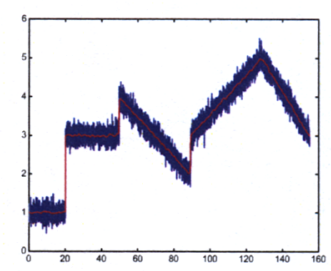

(c)

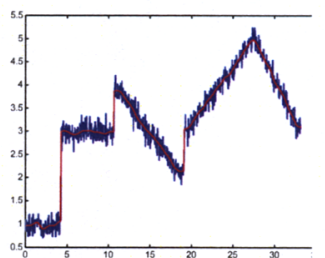

(h)

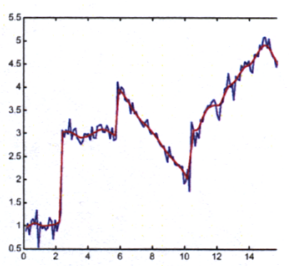

(m)

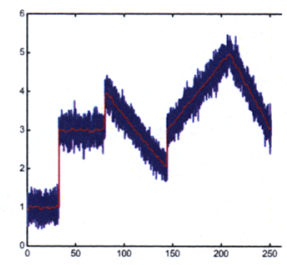

(d)

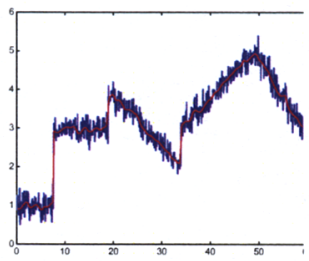

(i)

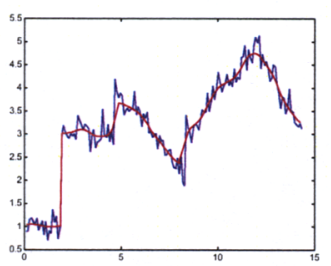

(n)

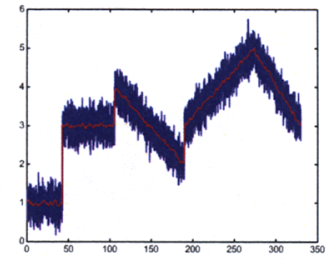

(e)

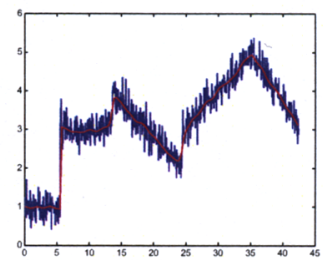

(j)

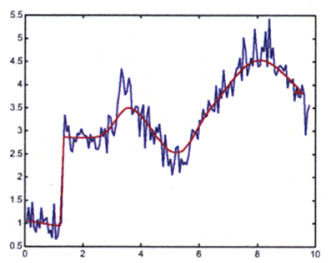

(o)

Fig. 5. Two realizations of the same piecewise smooth signal for two different SNR levels are shown in (a) and (b), along with their time difference histograms in (c) and (d).

[8] T. Hastie and W. Stuetzle, "Principal curves," Jour. Am. Statistical Assoc., vol. 84, pp. 502-516, 1989.

[9] Deniz Erdogmus and Umut Ozertem, "Self-consistent locally defined principal surfaces," in Proceedings of International Conference on Acoustics, Speech, and Signal Processing, 2007, pp. II549-II552.

[10] Umut Ozertem and Deniz Erdogmus, "Local conditions for critical and principal manifolds," in Proceedings of International Conference on Acoustics, Speech, and Signal Processing, 2008.

[11] Y. Cheng, "Mean shift, mode seeking, and clustering," IEEE Trans. on Pattern Analysis and Machine Intelligence, vol. 17, no. 8, pp. 790-799, 1995.

[12] Richard O. Duda, Peter E. Hart, and David G. Stork, Pattern Classification (2nd Edition), WileyInterscience, November 2000.

[13] B. W. Silverman, Density Estimation for Statistics and Data Analysis, Chapman \& Hall/CRC, April 1986.
[14] Emanuel Parzen, "On estimation of a probability density function and mode," The Annals of Mathematical Statistics, vol. 33, no. 3, pp. 1065-1076, 1962.

[15] Dorin Comaniciu, "An algorithm for data-driven bandwidth selection," IEEE Trans. Pattern Anal. Mach. Intell., vol. 25, no. 2, pp. 281-288, 2003.

[16] Lihi Zelnik-Manor and Pietro Perona, "Self-tuning spectral clustering.," in NIPS, 2004. 